
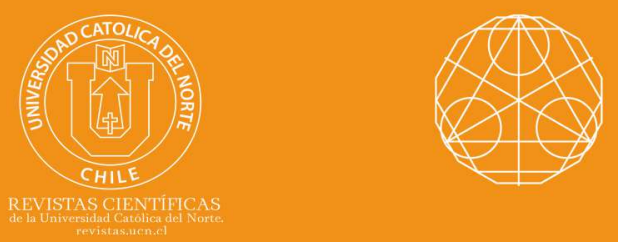

\title{
On the three families of extended Laguerre- based Apostol-type polynomials
}

\author{
M. A. Pathan ${ }^{1}$ (1) orcid.org/0000-0003-3918-7901 \\ Waseem A. Khan ${ }^{2}$ () orcid.org/0000-0002-4681-9885
}

${ }^{1}$ Centre for Mathematical and Statistical Sciences (CMSS), Thrissur, KL, India.

mapathan@gmail.com

${ }^{2}$ Prince Mohammad Bin Fahd University, Dept. of Mathematics and Natural Sciences, Al Khobar, Saudi Arabia.

ॠwhan1@pmu.edu.sa

Received: January 2020 | Accepted: June 2020

\section{Abstract:}

In this paper, we introduce a new class of generalized extended Laguerrebased Apostol-type-Bernoulli, Apostol-type-Euler and Apostoltype-Genocchi polynomials. These Apostol type polynomials are used to connect Fubini-Hermite and Bell-Hermite polynomials and to find new representations. We derive some implicit summation formulae and symmetric identities for these families of special functions by applying the generating functions.

Keywords: Gould-Hopper polynomials; Laguerre type exponential; Unified Apostol type polynomials; Extended Laguerre-based Apostol type polynomials; Fubini polynomials; Bell polynomials.

MSC (2020): 11B83, 11B37, 11B68, 33C45.

\section{Cite this article as (IEEE citation style):}

M. A. Pathan and W. A. Khan, "On the three families of extended Laguerre-based Apostol-type polynomials", Proyecciones (Antofagasta, On line), vol. 40, no. 2, pp. 313-334, 2021, doi: 10.22199/issn.0717-6279-2021-02-0019

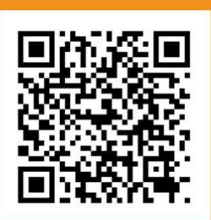

Article copyright: (C) 2021 M. A. Pathan and Waseem A. Khan. This is an open access article distributed under the terms of the Creative Commons License, which permits unrestricted use and distribution provided the original author and source are credited. 


\section{Introduction}

The generalization of 2-variable Kampé de Fériet polynomials (or, Gould Hopper polynomials) introduced by Gould and Hopper [15,p.58,(6.2)] (see also $[14,4])$ :

$$
H_{n}^{(p)}(x, y)=n ! \sum_{r=0}^{\left[\frac{n}{p}\right]} \frac{y^{r} x^{n-p r}}{r !(n-p r) !} .
$$

These polynomials are usually defined by the generating function $[15, \mathrm{p} .58,(6.3)]$ :

$$
e^{x t+y t^{p}}=\sum_{n=0}^{\infty} H_{n}^{(p)}(x, y) \frac{t^{n}}{n !},
$$

and reduce to the ordinary Hermite polynomials $H_{n}(x)$ (see $[1,2]$ ) when $p=2, y=-1$ and $x$ is replaced by $2 x$.

Recently, in the framework of the monomiality principle [8,13], a class of generalized exponential functions, the so-called Laguerre-type exponentials (shortly L-exponentials), was introduced by Dattoli and Ricci [14]. These functions are determined by using a differential isomorphism, denoted by the symbol $T:=T_{x}$, acting onto the space $A:=A_{x}$ of analytic functions of the $x$ variable by means of the correspondence

$$
D:=D_{x} \rightarrow D_{L}:=D x D ; \quad x \rightarrow D_{x}^{-1},
$$

where

$$
D_{x}^{-n}(1)=\frac{x^{n}}{n !},
$$

so that

$$
T\left(x^{n}\right)=\frac{x^{n}}{n !} .
$$

Note that the exponential function is transformed by $T$ into the Laguerrian exponential $e_{1}(x)$

$$
T e^{x}=e_{1}(x):=\sum_{n=0}^{\infty} \frac{x^{n}}{(n !)^{2}} .
$$

This is in accordance with the results of a paper by Dattoli and Ricci [11] about the definition of the higher-order Laguerre-type exponentials, which are defined in such a way that

$$
T_{x}^{s}\left(e^{x}\right)=e_{s}(x):=\sum_{k=0}^{\infty} \frac{x^{k}}{(n !)^{s+1}} .
$$


A first example of the above-mentioned isomorphism was found proving the connection between the Hermite-Kamp é de Fériet (or Gould-Hopper) polynomials $[7,10]$ and the two variable Laguerre polynomials. Now use of the above isomorphism already permitted us to extend the ordinary higher-order Laguerre polynomials which are the Laguerrian counterpart of the Gould-Hopper ones [6], and Laguerre type Bessel functions [9]. Particular cases of higher-order type Laguerre polynomials were used for the computation of moments of chaotic radiations (see [4]).

General classes of higher-order Laguerre polynomials were defined in [4] as

$$
L_{n}^{(j ; s, \sigma)}(x, y)=n ! \sum_{k=0}^{\left[\frac{n}{j}\right]} \frac{y^{k} x^{n-j k}}{(k !)^{\sigma+1}[(n-j k) !]^{s+1}},
$$

and are given by the generating function

$$
\sum_{n=0}^{\infty} L_{n}^{(j ; s, \sigma)}(x, y) \frac{t^{n}}{n !}=T_{x}^{s} T_{y}^{\sigma}\left(e^{x t+y t^{j}}\right)=e_{s}^{x t} e_{\sigma}^{y t^{j}} .
$$

Note that $L_{n}^{j}(x, y)$ are the 2-variable generalized Laguerre polynomials

$$
L_{n}^{j}(x, y)=n ! \sum_{k=0}^{\left[\frac{n}{j}\right]} \frac{y^{k} x^{n-j k}}{(k !)[(n-j k) !]}
$$

which are special cases of (1.3) and (1.4), when $s=\sigma=0$.

The exponential generating function for the geometric polynomials (also known as Fubini polynomials) $F_{n}(x)$ is given by [6] (see also [5])

$$
\frac{1}{1-x\left(e^{t}-1\right)}=\sum_{n=0}^{\infty} F_{n}(x) \frac{t^{n}}{n !} .
$$

Geometric polynomials also have close relation ship with Apostol-Bernoulli numbers $\beta_{n}(\lambda)$ and Euler numbers $E_{n}$ as (see [6])

$$
\begin{gathered}
\beta_{n}(\lambda)=\frac{n}{\lambda-1} F_{n}\left(\frac{\lambda}{1-\lambda}\right), \lambda \neq 1 \\
E_{n}=F_{n}\left(\frac{-1}{2}\right),
\end{gathered}
$$


where Apostol-Bernoulli numbers $\beta_{n}(\lambda)$ are defined by

$$
\left(\frac{t}{\lambda e^{t}-1}\right)=\sum_{n=0}^{\infty} \beta_{n}(\lambda) \frac{t^{n}}{n !} .
$$

Ramanujan obtained the exponential generating function of the exponential (one variable Bell) polynomials $\phi_{n}(x)$ (see [Berndt [3],Part 1, Chapter 3]) as

$$
e^{x\left(e^{t}-1\right)}=\sum_{n=0}^{\infty} \phi_{n}(x) \frac{t^{n}}{n !},
$$

and proved the recurrence relation

$$
\phi_{n+1}(x)=x\left(\phi_{n}(x)+\frac{d}{d x} \phi_{n}(x)\right) .
$$

Geometric and exponential polynomials are connected by the relation [6]

$$
F_{n}(x)=\int_{0}^{\infty} \phi_{n}(x) e^{-\lambda} d \lambda .
$$

Recently, Ozarslan [18] introduced the following unification of the ApostolBernoulli, Apostol-Euler and Apostol-Genocchi polynomials. Explicitly, Ozarslan studied the following generating function:

$$
\begin{array}{r}
f_{a, b}^{(\alpha)}(x ; t, a, b)=\left(\frac{2^{1-k} t^{k}}{\beta^{b} e^{t}-a^{b}}\right)^{\alpha} e^{x t}=\sum_{n=0}^{\infty} Y_{n, \beta}^{(\alpha)}(x ; k, a, b) \frac{t^{n}}{n !}, \\
\left(\left|t+b \ln \left(\frac{\beta}{\alpha}\right)\right|<2 \pi, k \in \mathbf{N}_{0} ; a, b \in \Re \backslash\{0\} ; \alpha, \beta \in \mathbf{C}\right) .
\end{array}
$$

For $\alpha=1$ in (1.8), we get

$$
\begin{gathered}
f_{a, b}(x ; t, a, b)=\frac{2^{1-k} t^{k}}{\beta^{b} e^{t}-a^{b}} e^{x t}=\sum_{n=0}^{\infty} Y_{n, \beta}(x ; k, a, b) \frac{t^{n}}{n !}, \\
\left(\left|t+b \ln \left(\frac{\beta}{\alpha}\right)\right|<2 \pi, k \in \mathbf{N}_{0} ; a, b \in \Re^{+} ; \beta \in \mathbf{C}\right) .
\end{gathered}
$$

From (1.8) and (1.9), we have

$$
Y_{n, \beta}^{(1)}(x ; k, a, b)=Y_{n, \beta}(x ; k, a, b),(n \in \mathbf{N}),
$$

which is defined by Ozden [19]. Ozden et al. [21] introduced many properties of these polynomials. We give some specific special cases: 
1. By substituting $a=b=k=1$ and $\beta=\lambda$ into (1.8), one has the ApostolBernoulli polynomials $Y_{n, \beta}^{(1)}(x ; 1,1,1)=B_{n}^{(\alpha)}(x ; \lambda)$, which are defined by means of the following generating function

$$
\left(\frac{t}{\lambda e^{t}-1}\right)^{\alpha} e^{x t}=\sum_{n=0}^{\infty} B_{n}^{(\alpha)}(x ; \lambda) \frac{t^{n}}{n !},(|t+\log \lambda|<2 \pi),
$$

(see for details [16-25]; see also the references cited in each of these earlier works).

For $\lambda=\alpha=1$ in (1.10), the result reduces to

$$
\frac{t}{e^{t}-1} e^{x t}=\sum_{n=0}^{\infty} B_{n}(x) \frac{t^{n}}{n !},|t|<2 \pi,
$$

where $B_{n}(x)$ denotes the classical Bernoulli polynomials (see from example [16-25]; see also the references cited in each of these earlier works).

2. If we substitute $b=\alpha=1, k=0, a=-1$ and $\beta=\lambda$ into (1.8), we have the Apostol-Euler polynomials $Y_{n, \lambda}^{(1)}(x ; 0,-1,1)=E_{n}^{(1)}(x, \lambda)$

$$
\left(\frac{2}{\lambda e^{t}+1}\right)^{\alpha} e^{x t}=\sum_{n=0}^{\infty} E_{n}^{(\alpha)}(x ; \lambda) \frac{t^{n}}{n !},(|t+\log \lambda|<\pi),
$$

(see for details [16-25]; see also the references cited in each of these earlier works).

For $\lambda=1$ in (1.11), the result reduces to

$$
\frac{2}{e^{t}+1} e^{x t}=\sum_{n=0}^{\infty} E_{n}(x) \frac{t^{n}}{n !},|t|<\pi,
$$

where $E_{n}(x)$ denotes the classical Euler polynomials (see from example [1625]; see also the references cited in each of these earlier works).

3. By substituting $b=\alpha=1, k=1, a=-1$ and $\beta=\lambda$ into (1.8), one has the Apostol-Genocchi polynomials $Y_{n, \beta}^{(1)}(x ; 1,-1,1)=\frac{1}{2} G_{n}(x ; \lambda)$, which is defined by means of the following generating function

$$
\left.\frac{2 t}{\lambda e^{t}+1} e^{x t}=\sum_{n=0}^{\infty} G_{n}(x ; \lambda) \frac{t^{n}}{n !},(|t+\log \lambda|<\pi)\right),
$$


(see for details [16-25]; see also the references cited in each of these earlier works).

4. By substituting $x=0$ in the generating function (1.8), we obtain the corresponding unification of the generating functions of Bernoulli, Euler and Genocchi numbers of higher order. Thus we have

$$
Y_{n, \beta}^{(\alpha)}(0 ; k, a, b)=Y_{n, \beta}^{(\alpha)}(k, a, b),(n \in \mathbf{N}) .
$$

Very recently, Pathan and Khan [22] introduced 2-variable Hermitebased Apostol type polynomials as follows:

Definition 1.1. The generalized Hermite-based Apostol type polynomials ${ }_{H} P_{n, \beta}^{(\alpha)}(x, y ; k, a, b, e)$ for nonnegative integer $n$ are defined by

$$
\begin{gathered}
\left(\frac{2^{1-k} t^{k}}{\beta^{b} e^{t}-a^{b}}\right)^{\alpha} e^{x t+y t^{2}}=\sum_{n=0}^{\infty} H_{n, \beta} P_{n,}^{(\alpha)}(x, y ; k, a, b, e) \frac{t^{n}}{n !}, \\
\left(\left|t+b \ln \left(\frac{\beta}{\alpha}\right)\right|<2 \pi, k \in \mathbf{N}_{0} ; a, b \in \Re \backslash\{0\} ; \alpha, \beta \in \mathbf{C}\right) .
\end{gathered}
$$

For the existence of the expansion, we need

(i) $|t|<2 \pi$ where $\alpha \in \mathbf{N}_{0}, k=1$ and $\left(\frac{\beta}{a}\right)^{b}=1 ;|t|<2 \pi$ when $\alpha \in \mathbf{N}_{0}$, $k=2,3, \cdots$ and $\left(\frac{\beta}{a}\right)^{b}=1 ;|t|<\left|b \log \left(\frac{\beta}{a}\right)\right|$ when $\alpha \in \mathbf{N}_{0}, k \in \mathbf{N}$ and $\left(\frac{\beta}{a}\right)^{b} \neq 1$ or $(\neq-1) ; x, y \in \mathbf{R}, \beta \in \mathbf{C} /\{0\}, 1^{\alpha}=1$.

(ii) $|t|<2 \pi$ when $\left(\frac{\beta}{a}\right)^{b}=-1 ;|t|<\left|b \log \left(\frac{\beta}{a}\right)\right|$ when $\left(\frac{\beta}{a}\right)^{b} \neq-1$, $x, y \in \mathbf{R}, k=0, \alpha, \beta \in \mathbf{C}, a, b, c \in \mathbf{C} 1^{\alpha}=1$.

(iii) $|t|<2 \pi$ when $\alpha \in \mathbf{N}_{0}$ and $\left(\frac{\beta}{a}\right)^{b}=-1, x, y \in \mathbf{R}, k \in \mathbf{N}, \beta \in \mathbf{C}$, $a, b, c \in \mathbf{C} /\{0\} 1^{\alpha}=1$ where $w=|w| e^{i \theta},-\pi \leq \theta<\pi$ and $\log (|w|)+i \theta$.

The generalized Stirling numbers of the second kinds $S(n, \nu, a, b, \beta)$ of order $\nu$ are defined in [26] as follows:

$$
\sum_{n=0}^{\infty} S(n, \nu, a, b, \beta) \frac{t^{n}}{n !}=\frac{\left(\beta^{b} e^{t}-a^{b}\right)^{\nu}}{\nu !} .
$$


Setting $\beta=\lambda, a=b=1$, (1.12) reduces to

$$
\sum_{n=0}^{\infty} S(n, \nu, \lambda) \frac{t^{n}}{n !}=\frac{\left(\lambda e^{t}-1\right)^{\nu}}{\nu !}
$$

\section{A new class of extended Laguerre-based Apostol-type poly- nomials}

In this section, we introduce a new class of extended Laguerre-based Apostoltype-Bernoulli, Apostol-type-Euler and Apostol-type-Genocchi polynomials

${ }_{L} Y_{n, \beta}^{(\alpha \mid j ; s, \sigma)}(x, y ; k, a, b)$, for a real or complex parameter $\alpha$ defined in a suitable neighborhood of $t=0$ by means of the following generating function

$$
\left(\frac{2^{1-k} t^{k}}{\beta^{b} e^{t}-a^{b}}\right)^{\alpha} e_{s}^{x t} e_{\sigma}^{y t^{j}}=\sum_{n=0}^{\infty}{ }_{L} Y_{n, \beta}^{(\alpha \mid j ; s, \sigma)}(x, y ; k, a, b) \frac{t^{n}}{n !},
$$

which contains as special cases not only generalized Apostol-type polynomials (1.8) to (1.11) but also generalization of the Laguerre polynomials $L_{n}^{(j ; s, \sigma)}(x, y)$ (c.f. Eq. $\left.(1.4)\right)$.

By substituting $x=y=0$ in (2.1), we obtain the corresponding unification of the generalized Apostol-type-Bernoulli, Apostol-type-Euler and Apostol-type-Genocchi numbers $Y_{n, \beta}^{(\alpha)}(k, a, b)$, for a real or complex parameter $\alpha$ given by means of the generating function

$$
\left(\frac{2^{1-k} t^{k}}{\beta^{b} e^{t}-a^{b}}\right)^{\alpha}=\sum_{n=0}^{\infty} Y_{n, \beta}^{(\alpha)}(k, a, b) \frac{t^{n}}{n !}
$$

Now by taking $s=\sigma=0$ in (2.1) and using (1.2), we have the representation

$$
{ }_{L} Y_{n, \beta}^{(\alpha \mid j ; 0,0)}(x, y ; k, a, b)=\sum_{r=0}^{n}\left(\begin{array}{l}
n \\
r
\end{array}\right) Y_{n-r, \beta}^{(\alpha)}(k, a, b) H_{r}^{j}(x, y) .
$$

It is evident that this explicit formula is a generalization of the following familiar results (see Kurt [16])

$$
B_{n}(x, y)=\sum_{r=0}^{n}\left(\begin{array}{l}
n \\
r
\end{array}\right) B_{n-r} H_{r}(x, y),
$$




$$
H_{n}(x, y)=\sum_{r=0}^{n}\left(\begin{array}{l}
n \\
r
\end{array}\right) \frac{1}{(n-r+1)} B_{r}(x, y) .
$$

On setting $j=2$ and $s=\sigma=0$ in (2.1), we get a recent result of Pathan and Khan [22,p.118, $\mathrm{Eq}(2.6)]$. For $k, \beta=1, a=b=1, j=2$ and $s=\sigma=0,(2.1)$ reduces to a known result of Pathan and Khan [23]. Further if $\alpha=1$ the result reduces to known result of Pathan [25], which is a generalization of the generating function of Dattoli et al. [12, p.386(1.6)] in the form

$$
\left(\frac{t}{e^{t}-1}\right) e^{x t+y t^{2}}=\sum_{n=0}^{\infty}{ }_{H} B_{n}(x, y) \frac{t^{n}}{n !} .
$$

Theorem 2.1. Let $a, b>0$. Then for $x, y \in \mathbf{R}$ and $n \geq 0$, we have

$$
\begin{gathered}
{ }_{L} Y_{n, \lambda}^{(\alpha \mid j ; s, \sigma)}(x, y ; 1,1,1)={ }_{L} B_{n}^{(\alpha \mid j ; s, \sigma)}(x, y ; \lambda), \\
{ }_{L} Y_{n, \lambda}^{(\alpha \mid j ; s, \sigma)}(x, y ; 0,1,-1)={ }_{L} E_{n}^{(\alpha \mid j ; s, \sigma)}(x, y ; \lambda), \\
{ }_{L} Y_{n, \frac{\lambda}{2}}^{(\alpha \mid j ; s, \sigma)}\left(x, y ; 1,-\frac{1}{2}, 1\right)={ }_{L} G_{n}^{(\alpha \mid j ; s, \sigma)}(x, y ; \lambda) .
\end{gathered}
$$

Proof. By using special values of $k, a, b$ in generating function (2.1), we can obtain the results (2.2). We omit the proof.

Theorem 2.2. Let $a, b>0$ and $a \neq b$. Then for $x, y \in \mathbf{R}$ and $n \geq 0$, we have

$$
\begin{gathered}
{ }_{L} Y_{n, \beta}^{(\alpha+\gamma \mid j ; s, \sigma)}(x+y, z+u ; k, a, b) \\
=\sum_{m=0}^{\infty}\left(\begin{array}{c}
n \\
m
\end{array}\right){ }_{L} Y_{m, \beta}^{(\gamma \mid j ; s, \sigma)}(y, u ; a, b){ }_{L} Y_{n-m, \beta}^{(\alpha \mid j ; s, \sigma)}(x, z ; k, a, b), \\
{ }_{L} Y_{n, \beta}^{(\alpha \mid j ; s, \sigma)}(x+z, y+u ; a, b) \\
=\sum_{n=0}^{m}\left(\begin{array}{c}
m \\
n
\end{array}\right){ }_{L} Y_{n-m, \beta}^{(\alpha \mid j ; s, \sigma)}(x, y ; k, a, b) L_{m}^{(j ; s, \sigma)}(z, u) .
\end{gathered}
$$


Proof. Applying definition (2.1), we have

$$
\begin{gathered}
\sum_{n=0}^{\infty}{ }_{L} Y_{n, \beta}^{(\alpha+\gamma \mid j ; s, \sigma)}(x+y, z+u ; k, a, b) \frac{t^{n}}{n !} \\
=\sum_{n=0}^{\infty}{ }_{L} Y_{n, \beta}^{(\alpha \mid j ; s, \sigma)}(x, z ; k, a, b) \frac{t^{n}}{n !} \sum_{m=0}^{\infty}{ }_{L} Y_{m, \beta}^{(\gamma \mid j ; s, \sigma)}(y, u ; k, a, b) \frac{t^{m}}{m !} \\
=\sum_{n=0}^{\infty} \sum_{m=0}^{n}{ }_{L} Y_{m, \beta}^{(\gamma \mid j ; s, \sigma)}(y, u ; k, a, b){ }_{L} Y_{n-m, \beta}^{(\alpha \mid j ; s, \sigma)}(x, z ; k, a, b) \frac{t^{n}}{(n-m) !} .
\end{gathered}
$$

Now equating the coefficients of the like powers of $t$ in the above equation, we get the result (2.3). Again by definition (2.1) of generalized polynomials, we have

$$
\begin{gathered}
\left(\frac{2^{1-k} t^{k}}{\beta^{b} e^{t}-a^{b}}\right)^{\alpha} e_{s}^{(x+z) t} e_{\sigma}^{(y+u) t^{j}} \\
=\sum_{n=0}^{\infty}{ }_{L} Y_{n, \beta}^{(\alpha \mid j ; s, \sigma)}(x+z, y+u ; k, a, b) \frac{t^{n}}{n !},
\end{gathered}
$$

which can be written as

$$
\begin{gathered}
\left(\frac{2^{1-k} t^{k}}{\beta^{b} e^{t}-a^{b}}\right)^{\alpha} e_{s}^{(x+z) t} e_{\sigma}^{(y+u) t^{j}} \\
=\sum_{n=0}^{\infty}{ }_{L} Y_{n, \beta}^{(\alpha \mid j ; s, \sigma)}(x, y ; k, a, b) \frac{t^{n}}{n !} \sum_{m=0}^{\infty} L_{m}^{(j ; s, \sigma)}(z, u) \frac{t^{m}}{m !} .
\end{gathered}
$$

On replacing $n$ by $n-m$ in the above equation and comparing the coefficients of $\frac{t^{n}}{n !}$, we get the desired result (2.4).

Theorem 2.3. For $n \geq 1$, we have

$$
\begin{gathered}
L_{n}^{(j ; s, \sigma)}(x, y) \\
=\frac{n !}{2^{1-k}(n+k) !}\left[\beta^{b}{ }_{L} Y_{n+k, \beta}^{(1,1 \mid j ; s, \sigma)}(x+1, y ; k, a, b)-a^{b}{ }_{L} Y_{n+k, \beta}^{(1,1 \mid j ; s, \sigma)}(x, y ; k, a, b)\right] .
\end{gathered}
$$


Proof. We begin with the definition (2.1) and write

$$
\begin{gathered}
e_{s}^{x t} e_{\sigma}^{y t^{j}}=\frac{1}{2^{1-k} t^{k}}\left(\frac{2^{1-k} t^{k}}{\beta^{b} e^{t}-a^{b}}\right)\left(\beta^{b} e^{t}-a^{b}\right) e_{s}^{x t} e_{\sigma}^{y t^{j}} \\
=\frac{1}{2^{1-k} t^{k}}\left[\left(\frac{2^{1-k} t^{k}}{\beta^{b} e^{t}-a^{b}}\right) \beta^{b} e_{s}^{(x+1) t} e_{\sigma}^{y t^{j}}-\left(\frac{2^{1-k} t^{k}}{\beta^{b} e^{t}-a^{b}}\right) a^{b} e_{s}^{x t} e_{\sigma}^{y t^{j}}\right] .
\end{gathered}
$$

Then using the definition of a general class of Laguerre polynomials $L_{n}^{(j ; s, \sigma)}(x, y)$ given by (1.9) together with (2.1), we have

$$
\begin{gathered}
\sum_{n=0}^{\infty} L_{n}^{(j ; s, \sigma)}(x, y) \frac{t^{n}}{n !} \\
=\sum_{n=0}^{\infty} \frac{1}{2^{1-k}(n+k) !}\left[\beta^{b}{ }_{L} Y_{n+k, \beta}^{(1,1 \mid j ; s, \sigma)}(x+1, y ; k, a, b)-a^{b}{ }_{L} Y_{n+k, \beta}^{(1,1 \mid j ; s, \sigma)}(x, y ; k, a, b)\right] t^{n} .
\end{gathered}
$$

Finally, comparing the coefficients of $t^{n}$, we get (2.5).

Theorem 2.4. The Hermite-based unified Apostol-type polynomials satisfying the following relation holds true:

$$
\begin{gathered}
\beta^{b}{ }_{H} Y_{n, \beta}^{(\alpha \mid j)}(x+1, y ; k, a, b)-a^{b}{ }_{H} Y_{n, \beta}^{(\alpha \mid j)}(x, y ; k, a, b) \\
=2^{1-k}{ }_{H} Y_{n-k, \beta}^{(\alpha-1 \mid j)}(x, y ; k, a, b) \frac{n !}{(n-k) !} .
\end{gathered}
$$

Proof. For $s=\sigma=0$ in the generating function (2.1), we have

$$
\begin{gathered}
\beta^{b}\left(\frac{2^{1-k} t^{k}}{\beta^{b} e^{t}-a^{b}}\right)^{\alpha} e^{(x+1) t+y t^{j}}-a^{b}\left(\frac{2^{1-k} t^{k}}{\beta^{b} e^{t}-a^{b}}\right)^{\alpha} e^{x t+y t^{j}} \\
=2^{1-k} t^{k} \sum_{n=0}^{\infty}{ }_{H} Y_{n, \beta}^{(\alpha-1 \mid j)}(x, y ; k, a, b) \frac{t^{n}}{n !}, \\
\sum_{n=0}^{\infty}\left(\beta_{H}^{b} Y_{n, \beta}^{(\alpha \mid j)}(x+1, y ; k, a, b)-a_{H}^{b} Y_{n, \beta}^{(\alpha \mid j)}(x, y ; k, a, b)\right) \frac{t^{n}}{n !} \\
=2^{1-k} \sum_{n=0}^{\infty}{ }_{H} Y_{n, \beta}^{(\alpha-1 \mid j)}(x, y ; k, a, b) \frac{t^{n+k}}{n !},
\end{gathered}
$$




$$
\begin{gathered}
\sum_{n=0}^{\infty}\left(\beta_{H}^{b} Y_{n, \beta}^{(\alpha \mid j)}(x+1, y ; k, a, b)-a_{H}^{b} Y_{n, \beta}^{(\alpha \mid j)}(x, y ; k, a, b)\right) \frac{t^{n}}{n !} \\
=2^{1-k} \sum_{n=k}^{\infty} H_{n-k, \beta}^{(\alpha-1 \mid j)}(x, y ; k, a, b) \frac{t^{n}}{(n-k) !} .
\end{gathered}
$$

By comparing the coefficients of $\frac{t^{n}}{n !}$, we arrive at the desired result (2.6).

Theorem 2.5. For $\alpha \in \mathbf{N}$, the following relation between the $\lambda$-Stirling numbers of second kinds and extended Laguerre-based unified Apostol type polynomials ${ }_{L} Y_{n, \beta}^{(\alpha \mid j ; s, \sigma)}(x, y ; k, a, b)$ holds true:

$$
\begin{aligned}
& a^{b \alpha} \alpha ! \sum_{r=0}^{n}\left(\begin{array}{c}
n \\
r
\end{array}\right){ }_{L} Y_{n-r, \beta}^{(\alpha \mid j ; s, \sigma)}(x, y ; k, a, b) S\left(r, \alpha,\left(\frac{\beta}{a}\right)^{b}\right) \\
& \quad=\left\{\begin{array}{cc}
0, & \text { for } n<k \alpha, \\
2^{(1-k) \alpha} L_{n-k \alpha}^{(j ; s, \sigma)}(x, y), & \text { for } n \geq k \alpha,
\end{array}\right.
\end{aligned}
$$

with $\alpha \in \mathbf{N}_{0}=N \cup\{0\}$ and $k \in \mathbf{N}$ fixed.

Proof. By using equations (2.1), (1.5) and (1.12), we have

$$
\begin{aligned}
& \sum_{n=0}^{\infty}{ }_{L} Y_{n, \beta}^{(\alpha \mid j ; s, \sigma)}(x, y ; k, a, b) \frac{t^{n}}{n !}=\left(\frac{2^{1-k} t^{k}}{\beta^{b} e^{t}-a^{b}}\right)^{\alpha} e_{s}^{x t} e_{\sigma}^{y t^{j}} \\
= & \frac{2^{(1-k) \alpha} t^{k \alpha}}{a^{b \alpha}\left(\left(\frac{\beta}{a}\right)^{b} e^{t}-1\right)^{\alpha}} e_{s}^{x t} e_{\sigma}^{y t^{j}}=\frac{2^{(1-k) \alpha} t^{k \alpha} e_{s}^{x t} e_{\sigma}^{y t^{j}}}{a^{b \alpha} \alpha ! \sum_{r=0}^{\infty} S\left(r, \alpha,\left(\frac{\beta}{a}\right)^{b}\right) \frac{t^{r}}{r !}},
\end{aligned}
$$

or, equivalently

$$
\begin{gathered}
\sum_{n=0}^{\infty}{ }_{L} Y_{n, \beta}^{(\alpha \mid j ; s, \sigma)}(x, y ; k, a, b) \frac{t^{n}}{n !} a^{b \alpha} \alpha ! \sum_{r=0}^{\infty} S\left(r, \alpha,\left(\frac{\beta}{a}\right)^{b}\right) \frac{t^{r}}{r !} \\
=2^{(1-k) \alpha} t^{k \alpha} \sum_{n=0}^{\infty} L_{n}^{(j ; s, \sigma)}(x, y) \frac{t^{n}}{n !}, \\
a^{b \alpha} \alpha ! \sum_{n=0}^{\infty} \sum_{r=0}^{n}\left(\begin{array}{c}
n \\
r
\end{array}\right){ }_{L} Y_{n-r, \beta}^{(\alpha \mid j ; s, \sigma)}(x, y ; k, a, b) S\left(r, \alpha,\left(\frac{\beta}{a}\right)^{b}\right) \frac{t^{n}}{n !}
\end{gathered}
$$




$$
=2^{(1-k) \alpha} \sum_{n=0}^{\infty} L_{n}^{(j ; s, \sigma)}(x, y) \frac{t^{n+k \alpha}}{n !} .
$$

By comparing the coefficients of $\frac{t^{n}}{n !}$, we obtain the desired result (2.7).

Theorem 2.6. The following relation between the $\lambda$-Stirling numbers of second kinds and extended Laguerre-based unified Apostol type polynomials ${ }_{L} Y_{n, \beta}^{(\alpha \mid j ; s, \sigma)}(x, y ; k, a, b)$ holds true:

$$
\begin{gathered}
a^{b \gamma} \gamma ! \sum_{r=0}^{n}\left(\begin{array}{c}
n \\
r
\end{array}\right){ }_{L} Y_{n-r, \beta}^{(\alpha \mid j ; s, \sigma)}(x, y ; k, a, b) S\left(r, \gamma,\left(\frac{\beta}{a}\right)^{b}\right) \\
\quad=\left\{\begin{array}{cc}
0, & \text { for } n<k \gamma, \\
2^{(k-1) \gamma} L_{n-k \gamma}^{(\alpha-\gamma \mid j ; s, \sigma)}(x, y), & \text { for } n \geq k \gamma,
\end{array}\right.
\end{gathered}
$$

with $\gamma \in \mathbf{N}_{0}=N \cup\{0\}$ and $k \in \mathbf{N}$ fixed.

Proof. From (2.1) and (1.12), we have

$$
\begin{gathered}
\sum_{n=0}^{\infty}{ }_{L} Y_{n, \beta}^{((\alpha-\gamma) \mid j ; s, \sigma)}(x, y ; k, a, b) \frac{t^{n}}{n !}=\left(\frac{2^{1-k} t^{k}}{\beta^{b} e^{t}-a^{b}}\right)^{\alpha-\gamma} e_{s}^{x t} e_{\sigma}^{y t^{j}} \\
=\left(\frac{2^{1-k} t^{k}}{\beta^{b} e^{t}-a^{b}}\right)^{\alpha} e_{s}^{x t} e_{\sigma}^{y t^{j}}\left(\frac{\beta^{b} e^{t}-a^{b}}{2^{1-k} t^{k}}\right)^{\gamma}, \\
2^{(k-1) \gamma} \sum_{n=0}^{\infty}{ }_{L} Y_{n, \beta}^{((\alpha-\gamma) \mid j ; s, \sigma)}(x, y ; k, a, b) \frac{t^{n+k \gamma}}{n !} \\
=a^{b \gamma} \gamma ! \sum_{n=0}^{\infty}{ }_{L} Y_{n, \beta}^{(\alpha \mid j ; s, \sigma)}(x, y ; k, a, b) \frac{t^{n}}{n !} \sum_{r=0}^{\infty} S\left(r, \gamma,\left(\frac{\beta}{a}\right)^{b}\right) \frac{t^{r}}{r !} .
\end{gathered}
$$

On comparing the coefficients of $\frac{t^{n}}{n !}$, we get the required result (2.8).

Theorem 2.7. The following implicit summation formulae for extended Laguerre-based Apostol-type polynomials ${ }_{L} Y_{n, \beta}^{(\alpha \mid j ; s, \sigma)}(x, y ; k, a, b)$ holds true: ${ }_{L} Y_{q+l, \beta}^{(\alpha \mid j ; s, \sigma)}(z, y ; k, a, b)=\sum_{n, p=0}^{q, l}\left(\begin{array}{l}q \\ n\end{array}\right)\left(\begin{array}{l}l \\ p\end{array}\right)(z-x)^{n+p}{ }_{L} Y_{q+l-p-n, \beta}^{(\alpha \mid j ; s, \sigma)}(x, y ; k, a, b)$. 
Proof. We replace $t$ by $t+u$ and rewrite the generating function (2.1) as

$$
\left(\frac{2^{1-k}(t+u)^{k}}{\beta^{b} e^{t+u}-a^{b}}\right)^{\alpha} e_{\sigma}^{y(t+u)^{j}}=e_{s}^{-x(t+u)} \sum_{q, l=0}^{\infty}{ }_{L} Y_{q+l, \beta}^{(\alpha \mid j ; s, \sigma)}(x, y ; k, a, b) \frac{t^{q}}{q !} \frac{u^{l}}{l !} .
$$

Replacing $x$ by $z$ in the above equation and equating the resulting equation to the above equation, we get

$$
e_{s}^{(z-x)(t+u)} \sum_{q, l=0}^{\infty}{ }_{L} Y_{q+l, \beta}^{(\alpha \mid j ; s, \sigma)}(x, y ; k, a, b) \frac{t^{q}}{q !} \frac{u^{l}}{l !}=\sum_{q, l=0}^{\infty}{ }_{L} Y_{q+l, \beta}^{(\alpha \mid j ; s, \sigma)}(z, y ; k, a, b) \frac{t^{q}}{q !} \frac{u^{l}}{l !} .
$$

On expanding exponential function (2.10) gives

$$
\begin{gathered}
\sum_{N=0}^{\infty} \frac{[(z-x)(t+u)]^{N}}{N !} \sum_{q, l=0}^{\infty}{ }_{L} Y_{q+l, \beta}^{(\alpha \mid j ; s, \sigma)}(x, y ; k, a, b) \frac{t^{q}}{q !} \frac{u^{l}}{l !} \\
=\sum_{q, l=0}^{\infty}{ }_{L} Y_{q+l, \beta}^{(\alpha \mid j ; s, \sigma)}(z, y ; k, a, b) \frac{t^{q}}{q !} \frac{u^{l}}{l !},
\end{gathered}
$$

which on using series manipulation formula

$$
\sum_{N=0}^{\infty} f(N) \frac{(x+y)^{N}}{N !}=\sum_{n, m=0}^{\infty} f(n+m) \frac{x^{n}}{n !} \frac{y^{m}}{m !},
$$

in the left hand side becomes

$$
\begin{gathered}
\sum_{n, p=0}^{\infty} \frac{(z-x)^{n+p} t^{n} u^{p}}{n ! p !} \sum_{q, l=0}^{\infty}{ }_{L} Y_{q+l, \beta}^{(\alpha \mid j ; s, \sigma)}(x, y ; k, a, b) \frac{t^{q}}{q !} \frac{u^{l}}{l !} \\
=\sum_{q, l=0}^{\infty}{ }_{H} L_{q+l, \beta}^{(\alpha \mid j ; s, \sigma)}(z, y ; k, a, b) \frac{t^{q}}{q !} \frac{u^{l}}{l !} .
\end{gathered}
$$

Now replacing $q$ by $q-n, l$ by $l-p$ in the left hand side of (2.11), we get

$$
\begin{gathered}
\sum_{q, l=0}^{\infty} \sum_{n, p=0}^{q, l} \frac{(z-x)^{n+p}}{n ! p !}{ }_{L} Y_{q+l-n-p, \beta}^{(\alpha \mid j ; s, \sigma)}(x, y ; k, a, b) \frac{t^{q}}{(q-n) !} \frac{u^{l}}{(l-p) !} \\
=\sum_{q, l=0}^{\infty}{ }_{L} Y_{q+l, \beta}^{(\alpha \mid j ; s, \sigma)}(z, y ; k, a, b) \frac{t^{q}}{q !} \frac{u^{l}}{l !} .
\end{gathered}
$$

Finally on equating the coefficients of the like powers of $t$ and $u$ in the above equation, we get the required result (2.9). 


\section{Extended Laguerre-based Apostol-type Bell and Fubini polynomials}

The starting point for the second generalization of the extended Laguerrebased Apostol-type polynomials, Bell polynomials and Gould-Hopper polynomials which we wish to consider is given by the following generating function

$$
\left(\frac{2^{1-k} t^{k}}{\beta^{b} e^{t}-a^{b}}\right)^{\alpha} e_{s}^{x\left(t+e^{t}-1\right)} e_{\sigma}^{y t^{j}}=\sum_{n=0}^{\infty}{ }_{L \phi} Y_{n, \beta}^{(\alpha \mid j ; s, \sigma)}(x, y ; k, a, b) \frac{t^{n}}{n !} .
$$

The clue to seeing a relation between Apostol-type polynomials, Bell polynomials [1,2] and extended Laguerre polynomials [1] such as (2.1), (1.7) and (1.5) and the special cases of (3.1) is given in the following useful Theorem.

Theorem 3.1. For $n \geq 0$, we have

$$
{ }_{\phi} L_{n, \beta}^{(\alpha, m \mid j ; s, \sigma)}(x, y ; k, a, b)=\sum_{r=0}^{n}\left(\begin{array}{c}
n \\
r
\end{array}\right) \phi_{r}^{(s)}(x)_{H} L_{n-r, \beta}^{(\alpha, m \mid j ; s, \sigma)}(x, y: k, a, b),
$$

where Bell polynomials $\phi_{n}(x)$ are given by (1.7).

Proof. We begin with the definition (3.1) and write

$$
\left(\frac{2^{1-k} t^{k}}{\beta^{b} e^{t}-a^{b}}\right)^{\alpha} e_{s}^{x\left(t+e^{t}-1\right)} e_{\sigma}^{y t^{j}}=e_{s}^{x\left(e^{t}-1\right)}\left(\frac{2^{1-k} t^{k}}{\beta^{b} e^{t}-a^{b}}\right)^{\alpha} e_{s}^{x t} e_{\sigma}^{y^{j}}
$$

Now the steps of the proof are similar to the proof of (2.4).

The third generalization of the Laguerre-based Apostol-type polynomials (2.1), Fubini polynomials (1.6) and Gould-Hopper polynomials (1.1) considered is given by the following generating function:

$$
\left(\frac{2^{1-k} t^{k}}{\beta^{b} e^{t}-a^{b}}\right)^{\alpha} \frac{e_{s}^{x t} e_{\sigma}^{y t^{j}}}{1-z\left(e^{t}-1\right)}=\sum_{n=0}^{\infty}{ }_{L F} Y_{n, \beta}^{(\alpha \mid j ; s, \sigma)}(x, y ; z ; k, a, b) \frac{t^{n}}{n !} .
$$

We are thus motivated to consider the following theorems on generalization of the Apostol type polynomials, Fubini polynomials and extended Laguerre polynomials. In the course of proving Theorems 3.2 and Theorem 3.3 , we have used the generating function (3.2). 
Theorem 3.2. For $n \geq 0$, we have

$$
{ }_{F} L_{n, \beta}^{(\alpha, m \mid j ; s, \sigma)}(x, y ; k, a, b)=\sum_{r=0}^{n}\left(\begin{array}{c}
n \\
r
\end{array}\right) F_{r}(z){ }_{L} Y_{n-r, \beta}^{(\alpha \mid j ; s, \sigma)}(x, y ; k, a, b),
$$

where Fubini polynomials $F_{n}(x)$ are given by (1.6).

Theorem 3.3. For $n \geq 0$, we have

$$
{ }_{L F} Y_{n, \beta}^{(\alpha \mid j ; s, \sigma)}(x, y ; z ; k, a, b)=\sum_{r=0}^{n}\left(\begin{array}{c}
n \\
r
\end{array}\right){ }_{L} F_{r}^{(j ; s, \sigma)}(x, y ; z) Y_{n-r, \beta}^{(\alpha)}(k, a, b),
$$

where extended Laguerre-based polynomials ${ }_{L} F_{n}^{(j ; s, \sigma)}(x, y ; z)$ are generated by

$$
\frac{e_{s}^{x t} e_{\sigma}^{y t^{j}}}{1-z\left(e^{t}-1\right)}=\sum_{n=0}^{\infty}{ }_{L} F_{n}^{(j ; s, \sigma)}(x, y ; z) \frac{t^{n}}{n !} .
$$

Interpreting the left hand side of (3.3) in terms of Fubini and extended Laguerre polynomials, we arrive at the representation

$$
{ }_{L} F_{n}^{(j ; s, \sigma)}(x, y ; z)=\sum_{r=0}^{n}\left(\begin{array}{c}
n \\
r
\end{array}\right) F_{r}(z) L_{n-r}^{(j ; s, \sigma)}(x, y) .
$$

Theorem 3.4. For $n \geq 0$, we have

$$
\begin{gathered}
{ }_{L F} Y_{n, \beta}^{(\alpha \mid j ; s, \sigma)}(x, y ; z ; k, a, b) \\
=\sum_{l=0}^{n}\left(\begin{array}{c}
n \\
l
\end{array}\right){ }_{L} Y_{n-l, \beta}^{(\alpha \mid j ; s, \sigma)}(x, y ; k, a, b) \sum_{k=0}^{l} z^{k} k ! S_{2}(l, k),
\end{gathered}
$$

where $S(n, \nu, 1)=S_{2}(n, \nu)$ is the Stirling number of second kind.

Proof. From (3.2), we have

$$
\begin{gathered}
\sum_{n=0}^{\infty}{ }_{L F} Y_{n, \beta}^{(\alpha \mid j ; s, \sigma)}(x, y ; z ; k, a, b) \frac{t^{n}}{n !}=\left(\frac{2^{1-k} t^{k}}{\beta^{b} e^{t}-a^{b}}\right)^{\alpha} \frac{e_{s}^{x t} e_{\sigma}^{y t^{j}}}{1-z\left(e^{t}-1\right)} \\
=\left(\frac{2^{1-k} t^{k}}{\beta^{b} e^{t}-a^{b}}\right)^{\alpha} e_{s}^{x t} e_{\sigma}^{y t^{j}} \sum_{k=0}^{\infty} z^{k}\left(e^{t}-1\right)^{k}
\end{gathered}
$$




$$
\begin{gathered}
=\left(\frac{2^{1-k} t^{k}}{\beta^{b} e^{t}-a^{b}}\right)^{\alpha} e_{s}^{x t} e_{\sigma}^{y t^{j}} \sum_{k=0}^{\infty} z^{k} \sum_{l=k}^{\infty} k ! S_{2}(l, k) \frac{t^{l}}{l !} \\
=\sum_{n=0}^{\infty}{ }_{L} Y_{n, \beta}^{(\alpha \mid j ; s, \sigma)}(x, y ; k, a, b) \frac{t^{n}}{n !} \sum_{l=0}^{\infty} z^{k} \sum_{k=0}^{l} k ! S_{2}(l, k) \frac{t^{l}}{l !} .
\end{gathered}
$$

Replacing $n$ by $n-l$ in above equation, we get

$$
\begin{aligned}
& \sum_{n=0}^{\infty} L F Y_{n, \beta}^{(\alpha \mid j ; s, \sigma)}(x, y ; z ; k, a, b) \frac{t^{n}}{n !} \\
& =\sum_{n=0}^{\infty}\left(\sum_{l=0}^{n}\left(\begin{array}{c}
n \\
l
\end{array}\right){ }_{L} Y_{n-l, \beta}^{(\alpha \mid j ; s, \sigma)}(x, y ; k, a, b) \sum_{k=0}^{l} z^{k} k ! S_{2}(l, k)\right) \frac{t^{n}}{n !} .
\end{aligned}
$$

Comparing the coefficients of $\frac{t^{n}}{n !}$ in both sides, we get (3.4).

Theorem 3.5. For $n \geq 0$, we have

$$
\begin{gathered}
{ }_{L F} Y_{n, \beta}^{(\alpha \mid j ; s, \sigma)}(x, y ; z ; k, a, b) \\
=\sum_{l=0}^{n}\left(\begin{array}{c}
n \\
l
\end{array}\right){ }_{L} Y_{n-l, \beta}^{(\alpha \mid j ; s, \sigma)}(x, y ; k, a, b) \sum_{k=0}^{l} z^{k} k ! S_{2}^{(s)}(l+r, k+r),
\end{gathered}
$$

where $S(n, \nu, 1)=S_{2}(n, \nu)$ is the Stirling number of second kind.

Proof. Replacing $x$ by $x+r$ in (3.2), we have

$$
\begin{gathered}
\sum_{n=0}^{\infty} L_{F} Y_{n, \beta}^{(\alpha \mid j ; s, \sigma)}(x, y ; z ; k, a, b) \frac{t^{n}}{n !} \\
=\left(\frac{2^{1-k} t^{k}}{\beta^{b} e^{t}-a^{b}}\right)^{\alpha} \frac{e_{s}^{(x+r) t} e_{\sigma}^{y t^{j}}}{1-z\left(e^{t}-1\right)} \\
=\left(\frac{2^{1-k} t^{k}}{\beta^{b} e^{t}-a^{b}}\right)^{\alpha} e_{s}^{x t} e_{\sigma}^{y t^{j}} e_{s}^{r t} \sum_{k=0}^{\infty} z^{k}\left(e^{t}-1\right)^{k} \\
=\left(\frac{2^{1-k} t^{k}}{\beta^{b} e^{t}-a^{b}}\right)^{\alpha} e_{s}^{x t} e_{\sigma}^{y t^{j}} e_{s}^{r t} \sum_{k=0}^{\infty} z^{k} \sum_{l=k}^{\infty} k ! S_{2}(l, k) \frac{t^{l}}{l !} \\
=\sum_{n=0}^{\infty}{ }_{L} Y_{n, \beta}^{(\alpha \mid j ; s, \sigma)}(x, y ; k, a, b) \frac{t^{n}}{n !} \sum_{l=0}^{\infty} z^{k} \sum_{k=0}^{l} k ! S_{2}^{(s)}(l+r, k+r) \frac{t^{l}}{l !} .
\end{gathered}
$$


Replacing $n$ by $n-l$ in above equation, we get

$$
\begin{gathered}
\sum_{n=0}^{\infty}{ }_{L F} Y_{n, \beta}^{(\alpha \mid j ; s, \sigma)}(x, y ; z ; k, a, b) \frac{t^{n}}{n !} \\
=\sum_{n=0}^{\infty}\left(\sum_{l=0}^{n}\left(\begin{array}{c}
n \\
l
\end{array}\right){ }_{L} Y_{n-l, \beta}^{(\alpha \mid j ; s, \sigma)}(x, y ; k, a, b) \sum_{k=0}^{l} z^{k} k ! S_{2}^{(s)}(l+r, k+r)\right) \frac{t^{n}}{n !} .
\end{gathered}
$$

Comparing the coefficients of $\frac{t^{n}}{n !}$ in both sides, we get (3.5).

\section{Symmetry identities}

In this section, we derive general symmetry identities for the extended Laguerre-based Apostol-type polynomials ${ }_{L} Y_{n, \beta}^{(\alpha \mid j ; s, \sigma)}(x, y ; k, a, b)$. We start with the following theorem.

Theorem 4.1. Let $\alpha, k \in \mathbf{N}_{0} ; a, b \in \mathbf{R} \backslash\{0\} ; \beta \in \mathbf{C}, x, y \in \mathbf{R}$ and $n \geq 0$, the following identity holds true:

$$
\begin{aligned}
& \sum_{r=0}^{n} d^{r} c^{n-r}\left(\begin{array}{c}
n \\
r
\end{array}\right){ }_{L} Y_{n-r, \beta}^{(\alpha \mid j ; s)}\left(d x, d^{2} y ; k, a, b\right)_{L} Y_{r, \beta}^{(\alpha \mid j ; s, \sigma)}\left(c x, c^{2} y ; k, a, b\right) \\
= & \sum_{r=0}^{n} c^{r} d^{n-r}\left(\begin{array}{c}
n \\
r
\end{array}\right){ }_{L} Y_{n-r, \beta}^{(\alpha \mid j ; s, \sigma)}\left(c x, c^{2} z ; k, a, b\right)_{L} Y_{r, \beta}^{(\alpha \mid j ; s, \sigma)}\left(d x, d^{2} y ; k, a, b\right) .
\end{aligned}
$$

Proof. Let

$$
f(t)=\left(\frac{2^{2(1-k)}(c d t)^{k}}{\left(\beta^{b} e^{c t}-a^{b}\right)\left(\beta^{b} e^{d t}-a^{b}\right)}\right)^{\alpha} e_{s}^{c d x t} e_{\sigma}^{c^{2} d^{2} y t^{j}} .
$$

Then the expression for $f(t)$ is symmetric in $c$ and $d$ and we can expand $f(t)$ into series in two ways to obtain

$$
\begin{aligned}
f(t) & =\sum_{n=0}^{\infty}{ }_{L} Y_{n, \beta}^{(\alpha \mid j ; s, \sigma)}\left(d x, d^{2} y ; k, a, b\right) \frac{(c t)^{n}}{n !} \sum_{r=0}^{\infty}{ }_{L} Y_{r, \beta}^{(\alpha \mid j ; s, \sigma)}\left(c x, c^{2} y ; k, a, b\right) \frac{(d t)^{r}}{r !} \\
f(t) & =\sum_{n=0}^{\infty}\left(\sum_{r=0}^{n} d^{r} c^{n-r}\left(\begin{array}{c}
n \\
r
\end{array}\right){ }_{L} Y_{n-r, \beta}^{(\alpha \mid j ; s, \sigma)}\left(d x, d^{2} y ; k, a, b\right){ }_{L} Y_{r, \beta}^{(\alpha \mid j ; s, \sigma)}\left(c x, c^{2} y ; k, a, b\right)\right) \frac{t^{n}}{n !}
\end{aligned}
$$


Similarly, we have

$$
f(t)=\sum_{n=0}^{\infty}\left(\sum_{r=0}^{n} c^{r} d^{n-r}\left(\begin{array}{c}
n \\
r
\end{array}\right){ }_{L} Y_{n-r, \beta}^{(\alpha \mid j ; s, \sigma)}\left(c x, c^{2} y ; k, a, b\right){ }_{L} Y_{r, \beta}^{(\alpha \mid j ; s, \sigma)}\left(d x, d^{2} y ; k, a, b\right)\right) \frac{t^{n}}{n !} .
$$

Comparing the coefficients $\frac{t^{n}}{n !}$ in last two equations, we get the desired result (4.1).

Theorem 4.2. The following symmetry relations for the extended Laguerrebased unified Apostol-type polynomials holds true;

$$
\begin{aligned}
& \sum_{m=0}^{c-1}\left(\frac{\beta}{a}\right)^{b m} \sum_{l=0}^{n}\left(\begin{array}{c}
n \\
l
\end{array}\right){ }_{H} Y_{n-l, \beta}^{(\alpha \mid j ; s, \sigma)}\left(d x, d^{2} z ; k, a, b\right) c^{n-k-l}(d m)^{l} \\
= & \sum_{m=0}^{d-1}\left(\frac{\beta}{a}\right)^{b m} \sum_{l=0}^{n}\left(\begin{array}{c}
n \\
l
\end{array}\right){ }_{H} Y_{n-l, \beta}^{(\alpha \mid j ; s, \sigma)}\left(c x, c^{2} z ; k, a, b\right) d^{n-k-l}(c m)^{l} .
\end{aligned}
$$

Proof. Let us consider

$$
\begin{gathered}
g(t)=\left(\frac{2^{1-k} t^{k}}{\beta^{b} e^{d t}-a^{b}}\right)^{\alpha} e_{s}^{c d x t} e_{\sigma}^{c^{2} d^{2} y t^{j}} \frac{\beta^{b d} e^{c d t}-a^{b d}}{\beta^{b} e^{c t}-a^{b}} \\
=\frac{1}{d^{k}}\left(\frac{2^{1-k} t^{k}}{\beta^{b} e^{d t}-a^{b}}\right)^{\alpha} e_{s}^{c d x t} e_{\sigma}^{c^{2} d^{2} y t^{j}} a^{b(d-1)}\left(\frac{1-\left(\frac{\beta}{a}\right)^{b d} e^{d c t}}{1-\left(\frac{\beta}{a}\right)^{b} e^{c t}}\right) \\
=a^{b(d-1)} d^{(-k)} \sum_{n=0}^{\infty}{ }_{L} Y_{n, \beta}^{(\alpha \mid j ; s, \sigma)}\left(c x, c^{2} z ; k, a, b\right) \frac{(d t)^{n}}{n !} \sum_{m=0}^{d-1}\left(\frac{\beta}{a}\right)^{b m} e^{c t m} \\
=a^{b(d-1)} d^{(-k)} \sum_{n=0}^{\infty}{ }_{L} Y_{n, \beta}^{(\alpha \mid j ; s, \sigma)}\left(c x, c^{2} z ; k, a, b\right) \frac{(d t)^{n}}{n !} \sum_{m=0}^{d-1}\left(\frac{\beta}{a}\right)^{b m} \sum_{l=0}^{\infty}(c m)^{l} \frac{t^{l}}{l !} \\
g(t)=a^{b(d-1)} d^{(-k)} \sum_{n=0}^{\infty} \sum_{m=0}^{d-1}\left(\frac{\beta}{a}\right)^{b m}\left(\sum_{l=0}^{n}\left(\begin{array}{l}
n \\
l
\end{array}\right)\right. \\
{ }_{L} Y_{n-l, \beta}^{(\alpha \mid j ; s, \sigma)}\left(c x, c^{2} z ; k, a, b\right) d^{n-l}(c m)^{l} \frac{l^{n}}{n !} .
\end{gathered}
$$

On the similar lines, we have

$$
g(t)=a^{b(d-1)} c^{(-k)} \sum_{n=0}^{\infty} \sum_{m=0}^{c-1}\left(\frac{\beta}{a}\right)^{b m}\left(\sum_{l=0}^{n}\left(\begin{array}{l}
n \\
l
\end{array}\right)\right.
$$




$$
{ }_{L} Y_{n-l, \beta}^{(\alpha \mid j ; s, \sigma)}\left(d x, d^{2} z ; k, a, b\right) c^{n-l}(d m) \frac{l^{l}}{n !} .
$$

On comparing the coefficients of $\frac{t^{n}}{n !}$ in (4.3) and (4.4), we arrive at the desired result (4.2).

Theorem 4.3. The extended Laguerre-based unified Apostol-type polynomials satisfy the following relation holds true:

$$
\begin{gathered}
\sum_{r=0}^{n}\left(\begin{array}{c}
n \\
r
\end{array}\right) c^{n-r} d^{r+1}{ }_{L} Y_{n-r, \beta}^{(\alpha \mid j ; s, \sigma)}(d x, d y ; k, a, b) \sum_{i=0}^{r}\left(\begin{array}{c}
r \\
i
\end{array}\right) S_{i}\left(c-1 ;\left(\frac{\beta}{a}\right)^{b}\right) \\
\times{ }_{L} Y_{r-i, \beta}^{(\alpha \mid j ; s, \sigma)}(c X, c Y ; k, a, b) \\
=\sum_{r=0}^{n}\left(\begin{array}{c}
n \\
r
\end{array}\right) d^{n-r} c^{r+1}{ }_{L} Y_{n-r, \beta}^{(\alpha \mid j ; s, \sigma)}(c x, c y ; k, a, b) \sum_{i=0}^{r}\left(\begin{array}{c}
r \\
i
\end{array}\right) S_{i}\left(d-1 ;\left(\frac{\beta}{a}\right)^{b}\right) \\
\times_{L} Y_{r-i, \beta}^{(\alpha \mid j ; s, \sigma)}(d X, d Y ; k, a, b) .
\end{gathered}
$$

Proof. We now use

$$
\begin{gathered}
h(t)=\left(\frac{2^{2(1-k)}(c d t)^{k}}{\left(\beta^{b} e^{d t}-a^{b}\right)\left(\beta^{b} e^{c t}-a^{b}\right)}\right)^{\alpha} e_{s}^{c d x t} e_{\sigma}^{c d y t^{j}} \frac{\beta^{b} e^{c d t}-a^{b}}{\beta^{b} e^{d t}-a^{b}} e_{s}^{c d X t} e_{\sigma}^{c d Y t^{j}} \\
=\sum_{n=0}^{\infty}{ }_{L} Y_{n, \beta}^{(\alpha \mid j ; s, \sigma)}(d x, d y ; k, a, b) \frac{(c t)^{n}}{n !} \sum_{i=0}^{\infty} S_{i}\left(c-1 ;\left(\frac{\beta}{a}\right)^{b}\right) \frac{(d t)^{n}}{n !} \\
\quad \times \sum_{r=0}^{\infty}{ }_{L} Y_{r, \beta}^{(\alpha \mid j ; s, \sigma)}(c X, c Y ; k, a, b) \frac{(d t)^{n}}{n !} \\
h(t)=\sum_{n=0}^{\infty} \sum_{r=0}^{n}\left(\begin{array}{c}
n \\
r
\end{array}\right) c^{n-r} d^{r+1}{ }_{L} Y_{n-r, \beta}^{(\alpha \mid j ; s, \sigma)}(d x, d y ; k, a, b) \sum_{i=0}^{r}\left(\begin{array}{c}
r \\
i
\end{array}\right)
\end{gathered}
$$

$\mathrm{S}_{i}\left(c-1 ;\left(\frac{\beta}{a}\right)^{b}\right) \times{ }_{L} Y_{r-i, \beta}^{(\alpha \mid j ; s, \sigma)}(c X, c Y ; k, a, b) \frac{t^{n}}{n !} \cdot(4.6)$

Using a similar plan, we get

$$
\begin{aligned}
h(t)= & \sum_{n=0}^{\infty} \sum_{r=0}^{n}\left(\begin{array}{c}
n \\
r
\end{array}\right) d^{n-r} c^{r+1}{ }_{L} Y_{n-r, \beta}^{(\alpha \mid j ; s, \sigma)}(c x, c y ; k, a, b) \sum_{i=0}^{r}\left(\begin{array}{c}
r \\
i
\end{array}\right) \\
& S_{i}\left(d-1 ;\left(\frac{\beta}{a}\right)^{b}\right) \times{ }_{L} Y_{r-i, \beta}^{(\alpha \mid j ; s, \sigma)}(d X, d Y ; k, a, b) \frac{t^{n}}{n !} .
\end{aligned}
$$

Equating the coefficients of $\frac{t^{n}}{n !}$ in last equations (4.6) and (4.7), we get (4.5). 


\section{References}

[1] L. C. Andrews, Special functions for engineers and mathematicians. New York, NY: Macmillan, 1985.

[2] E. T. Bell, "Exponential polynomials", Annals of mathematical, vol. 35, no. 2, pp. 258-277, 1934, doi: 10.2307/ 1968431

[3] B. C. Berndt, Ramanujan's notebooks, vol. 1. New York, NY: Springer, 1985, doi: 10.1007/978-1-4612-1088-7

[4] A. E. Bernarini, G. K. Dattoli, and P. A. Ricci, "L-exponentials and higher order Laguerre polynomials", in Proceeding of the Fourth Annual Conference of the Society for Special Functions and their Applications, Jaipur (India), March 4-6, 2003.

[5] K. N. Boyadzhiev, "A series transformation formula and related polynomials", International journal mathematical sciences, vol. 2005, Art ID. 792107, 2005, doi: 10.1155/ IJMMS.2005.3849

[6] K. N. Boyadzhiev, "Apostol-Bernoulli functions, derivative polynomials and Eulerian polynomials", Advances and applications in discrete mathematics, vol. 1, no. 2, pp. 109-122, 2008. [On line]. Available: https:/ / bit.ly/ 3rqe1Z7

[7] G. Bretti, C. Cesarano, and P. Ricci, "Laguerre-type exponentials and generalized Appell polynomials", Computers \& mathematics with applications, vol. 48, no. 5-6, pp. 833-839, 2004, doi: 10.1016/j.camwa.2003.09.031

[8] Y. B. Cheikh, "Some results on quasi-monomiality", Applied mathematics and computation, vol. 141, no. 1, pp. 63-76, 2003, doi: 10.1016/ s0096-3003(02)00321-1

[9] C. Cesarano, B. Germano, and P. E. Ricci, "Laguerre-type Bessel functions", Integral transforms and special functions, vol. 16, no. 4, pp. 315-322, 2005, doi: 10.1080/ 10652460412331270629

[10] G. Dattoli and P. E. Ricci, "Multi-index polynomials and applications to statistical problems", Nuovo Cimento della Societa Italiana di Fisica B, vol. 118, no. 6, pp. 625-633, 2003. 
[11] G. Dattoli, A. Arena, P. E. and Ricci, "Laguerrian eigenvalues problems and Wright functions", Mathematical computer modeling, vol. 40, no. 7-8, pp. 877-881 , 2004, doi: 10.1016/j.mcm.2004.10.017

[12] G. Dattoli, S. Lorenzutta, and C. Cesarano, "Finite sums and generalized forms of Bernoulli polynomials", Rendiconti di mathematica, vol. 19, pp. 385-391, 1999.

[13] G. Dattoli, "Hermite-Bessel and Laguerre-Bessel functions: a by-product of the monomiality principle," in Proceeding. of the Workshop on Advanced Special Functions and Applications, Melfi (PZ) Italia 9-12 May 1999, 2006, pp. 147-164, doi: 10.4399/97888799926578

[14] G. Dattoli and P. E. Ricci, "Laguerre-type exponentials and the relevant L-circular and L-hyperbolic functions", Georgian mathematical journal, vol. 10, no. 3, 2003, pp. 481-494, 2003, doi: 10.1515/ GMJ.2003.481

[15] H. W. Gould and A. T. Hopper, "Operational formulas connected with two generalizations of Hermite polynomials", Duke mathematical journal, vol. 29, no. 1, 1962, pp. 51-62, doi: 10.1215/ S0012-7094-6202907-1

[16] B. Kurt, "A further generalization of Bernoulli polynomials and on the 2D-Bernoulli polynomials $\mathrm{B}_{\mathrm{n}}^{2}(\mathrm{x}, \mathrm{y})$ ", Applied mathematical sciences, vol. 4, no. 47, pp. 2315-2322, 2010. [On line]. Available: https:/ / bit.ly/ 36HyKPZ

[17] M. A. Özarslan, "Hermite-based unified Apostol-Bernoulli, Euler and Genocchi polynomials", Advances differential equations, vol. 2013, Art ID. 116, 2013, doi: 10.1186/ 1687-1847-2013-116

[18] M. A. Özarslan, “Unified Apostol-Bernoulli, Euler and Genocchi polynomials", Computer mathematical applied, vol. 62, no. 1, pp. 2452-2462, 2011, doi: 10.1016/j.camwa.2011.07.031

[19] H. Özden, "Unification of generating functions of the Bernoulli, Euler and Genocchi numbers and polynomials", in ICNAAM 2010: International Conference of Numerical Analysis and Applied Mathematics 2010, vol. 1281, T. E. Simos, G. Psihoyios, and C. Tsitouras, Eds. 2010, doi: 10.1063/ 1.3497848

[20] H. Özden, "Generating function of the unified representation of the Bernoulli, Euler and Genocchi polynomials of higher order", in Numerical analysis and applied mathematics ICNAAM 2011: International Conference on Numerical Analysis and Applied Mathematics, vol. 1389, T. E. Simos, G. Psihoyios, C. Tsitouras, and Z. Anastassi, Eds. 2011, doi: 10.1063/ 1.3636736 
[21] H. Özden, Y. Simsek, and H. Srivastava, "A unified presentation of the generating functions of the generalized Bernoulli, Euler and Genocchi polynomials", Computers \& mathematics with applications, vol. 60, no. 10, pp. 2779-2787, 2010, doi: 10.1016/j.camwa.2010.09.031

[22] M. A. Pathan and W. Khan, "Some implicit summation formulas and symmetric identities for the generalized Hermite-Based polynomials", Acta Universitatis Apulensis, vol. 39, pp. 113-136, 2014, doi: 10.17114/j.aua.2014.39.11

[23] M. A. Pathan and W. A. Khan, "Some implicit summation formulas and symmetric identities for the generalized Hermite-Bernoulli polynomials", Mediterranean journal of mathematics, vol. 12, no. 3, pp. 679-695, 2014, doi: 10.1007/ s00009-014-0423-0

[24] M. A. Pathan and W. A. Khan, "A new class of generalized polynomials associated with Hermite and Euler polynomials", Mediterranean journal of mathematics, vol. 13, no. 3, pp. 913-928, 2015, doi: $10.1007 / \mathrm{s} 00009-015-0551-1$

[25] M. A. Pathan, "A new class of generalized Hermite-Bernoulli polynomials", Georgian mathematical journal, vol. 19, no. 3, pp. 559-573, 2012, doi: $10.1515 /$ gmj-2012-0019

[26] Y. Simsek, "Generating functions for generalized Stirling type numbers, array type polynomials, Eulerian type polynomials and their applications", Fixed point theory and applications, vol. 2013, Art ID. 87, 2013, doi: 10.1186/ 1687-1812-2013-87 